\title{
Developmental vitamin $D$ and autism spectrum disorders: findings from the Stockholm Youth Cohort
}

\author{
Brian K. Lee $\mathbb{1}^{1,2,3} \cdot$ Darryl W. Eyles ${ }^{4,5} \cdot$ Cecilia Magnusson $^{3} \cdot$ Craig J. Newschaffer $^{1,2} \cdot$ John J. McGrath $\mathbb{D}^{4,5,6}$. \\ David Kvaskoff $^{4} \cdot$ Pauline Ko $^{4,5} \cdot$ Christina Dalman $^{3} \cdot$ Håkan Karlsson $^{7} \cdot$ Renee M. Gardner ${ }^{3}$
}

Received: 5 June 2019 / Revised: 7 October 2019 / Accepted: 23 October 2019 / Published online: 6 November 2019

(c) The Author(s) 2019. This article is published with open access

\begin{abstract}
Animal studies indicate that early life vitamin D is crucial for proper neurodevelopment. Few studies have examined whether maternal and neonatal vitamin D concentrations influence risk of autism spectrum disorders (ASD). Participants were sampled from the Stockholm Youth Cohort, a register-based cohort in Sweden. Concentrations of total 25-hydroxyvitamin D (25OHD) were assessed from maternal and neonatal biosamples using a highly sensitive liquid chromatography tandem mass spectrometry method. The maternal sample consisted of 449 ASD cases and 574 controls, the neonatal sample: 1399 ASD cases and 1607 controls; and the paired maternal-neonatal sample: 340 ASD cases and 426 controls. Maternal 25OHD was not associated with child ASD in the overall sample. However, in Nordic-born mothers, maternal 25OHD insufficiency $(25-<50 \mathrm{nmol} / \mathrm{L})$ at $\sim 11$ weeks gestation was associated with 1.58 times higher odds of ASD $(95 \%$ CI: $1.00,2.49)$ as compared with $25 \mathrm{OHD}$ sufficiency $(\geq 50 \mathrm{nmol} / \mathrm{L})$. Neonatal $25 \mathrm{OHD}<25 \mathrm{nmol} / \mathrm{L}$ was associated with 1.33 times higher odds of ASD (95\% CI: $1.02,1.75$ ) as compared with $25 \mathrm{OHD} \geq 50 \mathrm{nmol} / \mathrm{L}$. Sibling-matched control analyses indicated these associations were not likely due to familial confounding. Children with both maternal 25OHD and neonatal 25OHD below the median had 1.75 (95\% CI: 1.08, 2.86) times the odds of ASD compared with children with maternal and neonatal $25 \mathrm{OHD}$ both below the median. Our results are consistent with an increasing body of evidence suggesting that vitamin D concentrations in early life may be associated with increased risk of neurodevelopmental disorders including ASD.
\end{abstract}

Supplementary information The online version of this article (https:// doi.org/10.1038/s41380-019-0578-y) contains supplementary material, which is available to authorized users.

Brian K. Lee

bk129@drexel.edu

1 Department of Epidemiology and Biostatistics, Drexel University School of Public Health, Philadelphia, PA, USA

2 A.J. Drexel Autism Institute, Philadelphia, PA, USA

3 Department of Public Health Sciences, Karolinska Institutet, Stockholm, Sweden

4 Queensland Brain Institute, University of Queensland, St. Lucia, QLD, Australia

5 Queensland Centre for Mental Health Research, The Park Centre for Mental Health, Wacol, QLD, Australia

6 National Centre for Register-based Research, Aarhus BSS, Aarhus University, Aarhus, Denmark

7 Department of Neuroscience, Karolinska Institutet, Stockholm, Sweden

\section{Introduction}

Early life nutrition is critical for proper neurodevelopment [1] and the paradigm may extend to autism spectrum disorders (ASD) as well [2]. Epidemiological studies have suggested potential links between multiple nutritional factors and ASD risk, including antenatal folic acid [3] or multivitamin supplementation [4], fat intake [5], and iron intake [6], although findings are not consistent across studies [7, 8]. One nutritional factor of potential relevance to ASD risk is vitamin D [9]. Although commonly referred to as the "sunshine vitamin", vitamin D is a neuroactive hormone that is required for normal brain homeostasis and neurodevelopment [10]. Most ( 90\%) vitamin $\mathrm{D}$ is synthesized in response to skin exposure to UV-B light with the rest derived from dietary sources [11]. Vitamin D deficiency and insufficiency are common globally, especially in pregnant women [12,13], and are more prevalent in winter, in high latitudes, urban settings and in dark-skinned individuals $[14,15]$. 
Recent epidemiological studies have provided evidence that lower concentrations of gestational 25-hydroxyvitamin D (25OHD) may be correlated with increased risk of ASD phenotypes. In an Australian birth cohort, maternal midgestation 25OHD insufficiency was associated with increased offspring risk of high scores on the Attention Switching subscale of the Autism Spectrum Quotient [16] and increased risk of language impairment [17]. In the Generation R study in the Netherlands, lower mid-gestation and cord blood 25OHD concentrations were associated with more abnormal scores on the Social Responsiveness Scale, a measure of an ASD-related phenotype [18]. There is also evidence suggesting that early life 25OHD may be associated with ASD case status, although findings have been mixed. Two studies-one of Swedish siblings with ASD and their unaffected siblings ( $n=58$ pairs) and a Chinese ASD case-control study ( $n=68$ cases) -found that lower levels of neonatal [19] and 1st trimester 25OHD [20] were associated with increased odds of ASD. In the Generation R study (also based on 68 cases), mid-gestation vitamin $\mathrm{D}$ deficiency was associated with 2.4 times higher risk of ASD [21]. However, two recent studies in California found that neonatal 25OHD was not associated with ASD overall, although there was some evidence of associations in subgroups (see Discussion) [22, 23].

In the present study, we examine the associations of maternal and neonatal 25OHD and later risk of ASD in a Swedish population-based sample. Because of prior reported associations of early life $25 \mathrm{OHD}$ with intellectual function, we also explored whether associations were different for ASD subtyped by presence or absence of cooccurring intellectual disability (ID).

\section{Methods}

\section{Study sample}

Ethical approval was provided by the regional ethical review board for Karolinska Institutet (DNR 2010/1185-31/ $5)$. As this was a register-based study, no individual level consent was required. All data were anonymized before being received by the research team. The Stockholm Youth Cohort (SYC) is a register-based cohort of all children aged 0-17 years living in Stockholm County. The source population for the present study was the 98,597 SYC participants born in Sweden 1996-2000 and resident for at least 4 years in Stockholm County through 2011. The length of residency exclusion criteria is to allow a reasonable opportunity for local healthcare services to detect ASD.

There were four analytic samples based on the available biospecimens (Figs. S1 and S2): the maternal sample examining prenatal 25OHD in maternal sera; the neonatal sample examining neonatal 25OHD in dried blood spots (DBS) taken soon after birth; the neonatal sibling sample examining neonatal 25OHD in cases matched to unaffected siblings; and the paired maternal-neonatal sample examining both maternal and neonatal 25OHD (restricted to persons with both maternal and neonatal 25OHD measures).

\section{ASD diagnoses}

Developmental screenings are carried out free of charge in Stockholm County at regular intervals from 1 to 60 months by a pediatrician or nurse. With a positive screen, children are referred to specialist services for formal diagnostic assessment. We implemented a register-based case-finding procedure covering all pathways to care in Stockholm County. Briefly, ASD status captured by ICD-9 (299), ICD10 (F84), and DSM-IV (299) codes using the National Patient Register, Habilitation Register, the Clinical Database for Child and Adolescent Psychiatry, and the VAL database covering all inpatient and outpatient health services use in Stockholm County. Ascertainment of ID was based on ICD-9 (317-319), ICD-10 (F70-F79), and DSMIV (317-319) codes and supplemented with information in the Habilitation Register, which classifies services recipients as having ID or not. In a validation study, we found case-notes consistent with an ASD diagnosis for $96.0 \%$ of the register-identified cases [24].

\section{HD analysis}

Since 1975 in Sweden, DBS have been collected from all newborns at $\sim 3-5$ days of age and stored at a central biobank for metabolic disease screening. DBS were obtained for each participant, and $3.2 \mathrm{~mm}$ punches were extracted and derivatized in a process optimized for detection of vitamin D [25]. For the maternal sample, sera were obtained at median 10.9 weeks gestation (IQR: 9.3, 13.0). In total, $78.0 \%$ of sera samples were from the 1 st trimester, $19.0 \%$ from the 2nd, and $3.0 \%$ from the 3rd trimester. Because of the range of gestational ages at which sera was collected, and because $25 \mathrm{OHD}$ varies across the calendar year (i.e., seasonal variation) and during pregnancy, 25OHD values are not directly comparable between participants if, for example, one participant had a 3rd trimester 25OHD measurement while another participant had a 1st trimester measurement. Consistent with recent practice [26], we used cosinor modeling to standardize 25OHD values at gestational age of 10.9 weeks (Supplement Fig. S3).

25OHD was measured using a tandem mass spectrometry assay developed by DWE [25], including recent methods to improve sensitivity to $100 \mathrm{pmol} / \mathrm{L}$ in DBS [27]. This assay separately measures the endogenous 25OHD3 and any exogenous 25OHD2 resulting from maternal 
supplementation. This assay has been used in a number of studies exploring the association between 25OHD with autism and schizophrenia [18, 21-23, 28, 29]. The analytic variable of interest was the concentration of total $250 \mathrm{HD}$ $(25 \mathrm{OHD} 3+25 \mathrm{OHD} 2)$. These values largely represent the measurement of $25 \mathrm{OHD} 3$, as few serum $(0.2 \%)$ or DBS samples $(0.4 \%)$ had detectable levels of 25OHD2. Because the neonatal samples were obtained from venous blood, the following correction formula was used to estimate equivalent serum levels: (DBS 25OHD3 + DBS 25OHD2) $\times(1 /$ $(1-0.55))$, where the 0.55 is a reference hematocrit value for venous blood [25, 30-32].

To assess construct validity of the $250 \mathrm{HD}$ measures, we assessed (1) whether there was seasonal variation in 250HD depending on month of collection and (2) whether 250HD measures correlated with sunlight intensity around the time of birth. Sunlight measurements for Stockholm during the study period (specifically, average insolation incident on a horizontal surface) were obtained from the NASA Prediction of Worldwide Energy Resource [33]. To standardize comparisons, 25OHD values and solar radiation values were $\mathrm{z}$-transformed and then plotted against each other.

\section{Covariates}

In addition to the aforementioned health registers, covariate data were extracted from the Medical Birth Register and the Integrated Database for Labor Market Research. Covariates were identified a priori from the literature as being associated with either vitamin D or ASD status. Year and season of birth were always included to account for calendar time and seasonal trends [34]. Other covariates considered included diagnoses of maternal autism, ID, and other psychiatric disorders [35] and maternal health variables assessed at the first antenatal visit, including body mass index [36], smoking [37], and nutritional supplement use [4]. Gestational age at birth was included to account for the increased risk of ASD observed for non-term births [38]. Although race/ethnicity data were not available, maternal region of origin (Nordic, other Europe, Africa, Asia, other) was included in models.

\section{Statistical analysis}

The outcomes of interest were ASD, ASD without cooccurring ID, and ASD with ID. Logistic regression models were used to estimate odds ratios and 95\% confidence intervals (OR, 95\% CI). 25OHD was analyzed with the following categorizations: deficient $(<25 \mathrm{nmol} / \mathrm{L})$, insufficient $(25-<50 \mathrm{nmol} / \mathrm{L})$, and sufficient $(\geq 50 \mathrm{nmol} / \mathrm{L})$, consistent with recommendations set by the Institute of Medicine [39]. To check for potential threshold or nonlinear associations, penalized cubic regression smoothing splines in generalized additive models were implemented to assess 25OHD as a continuous variable. We examined possible effect modification of the associations of neonatal vitamin $\mathrm{D}$ and $\mathrm{ASD}$ by child sex and by maternal region of origin as a proxy for skin pigmentation. For the sibling-matched sample, conditional logistic regression was employed to account for the matched nature of the analysis. Because siblings share genetics and early life environment, this analysis can test whether results from the standard analyses may be due to confounding from these sources.

Biosamples were randomly plated to avoid the possibility of systematic confounding due to plate effects. To maintain the blinded status of lab personnel as well as participant integrity, unaffected siblings were plated separately from their matched cases. Therefore, the plate-specific 25OHD zscore (calculated as the number of standard deviations away from the plate-specific mean a particular observation is) was used for the matched sibling analysis. All analyses were conducted using $\mathrm{R}$ 3.4.1 [40]. $\mathrm{R}$ code for analysis will be made available upon request.

Although predictive modeling for standardization of $25 \mathrm{OHD}$ is common, inferences resulting from this modeling may be incorrect if the underlying predictive model used to standardize the values is incorrect. We conducted a sensitivity analysis restricted to the $78 \%$ of participants with $25 \mathrm{OHD}$ values measured in the 1 st trimester, using the natural 25OHD values instead of the standardized 25OHD values.

\section{Results}

\section{Study sample}

The maternal sample consisted of 449 ASD cases and 574 controls; the neonatal sample, 1399 ASD cases and 1607 controls; the neonatal sibling sample, 357 ASD cases matched to 364 unaffected siblings; and the paired maternalneonatal sample, 340 ASD cases and 426 controls. Because the samples were broadly similar, the neonatal sample is described here, and the other samples are described in Table S1. Of the neonatal controls, 25OHD-sufficient participants were more likely to have mothers who took multivitamin supplements, who had a normal BMI, and who were born in a Nordic country, compared with insufficient or deficient participants (Table 1). Persons excluded due to non-collection of samples and laboratory failures differed from persons included in the sample on some aspects. They were more likely to be born in spring; have missing maternal BMI; less maternal prenatal iron only supplement use; and less likely to have maternal origin in Nordic countries (Table S2). 
Table 1 Neonatal analytic sample characteristics

\begin{tabular}{|c|c|c|c|c|c|}
\hline & \multicolumn{2}{|c|}{ By case status } & \multicolumn{3}{|c|}{ By 25OHD status (No ASD only) } \\
\hline & $\begin{array}{l}\text { No ASD: } \\
n=1607\end{array}$ & $\begin{array}{l}\text { ASD: } \\
n=1399\end{array}$ & $\begin{array}{l}\text { 25OHD Sufficient } \geq \\
50 \mathrm{nmol} / \mathrm{L}, n=186\end{array}$ & $\begin{array}{l}\text { 25OHD: Insufficient } \\
25-<50 \mathrm{nmol} / \mathrm{L}, n=682\end{array}$ & $\begin{array}{l}\text { 25OHD: Deficient } \\
<25 \mathrm{nmol} / \mathrm{L}, n=739\end{array}$ \\
\hline \multicolumn{6}{|l|}{ Outcome } \\
\hline ASD no ID & - & $947(67.7)$ & - & - & - \\
\hline ASD with ID & - & $452(32.3)$ & - & - & - \\
\hline No ASD & $1607(100)$ & & $186(100)$ & $682(100)$ & $739(100)$ \\
\hline \multicolumn{6}{|l|}{ Season of birth } \\
\hline Spring (Mar-May) & $407(25.3)$ & $304(21.6)$ & $22(11.8)$ & $148(21.7)$ & $237(32.1)$ \\
\hline Summer (Jun-Aug) & $436(27.1)$ & $405(28.9)$ & $103(55.4)$ & $193(28.3)$ & $140(18.9)$ \\
\hline Fall (Sep-Nov) & $391(24.3)$ & $376(26.9)$ & $47(25.3)$ & $185(27.1)$ & $159(21.5)$ \\
\hline Winter (Dec-Feb) & $373(23.2)$ & $314(22.4)$ & $14(7.5)$ & $156(22.9)$ & $203(27.5)$ \\
\hline Male & $819(51.0)$ & $1064(76.1)$ & $85(45.7)$ & $365(53.5)$ & $369(49.9)$ \\
\hline Maternal age, mean (SD) & $30.2(5.1)$ & $30.0(5.3)$ & $30.7(4.6)$ & $30.8(4.9)$ & $29.5(5.2)$ \\
\hline \multicolumn{6}{|l|}{ Maternal BMI } \\
\hline Normal & $828(51.5)$ & $623(44.5)$ & $107(57.5)$ & $348(51.0)$ & $373(50.5)$ \\
\hline Underweight & $38(2.4)$ & $37(2.6)$ & $2(1.1)$ & $17(2.5)$ & $19(2.6)$ \\
\hline Overweight & $262(16.3)$ & $260(18.6)$ & $20(10.8)$ & $108(15.8)$ & $134(18.1)$ \\
\hline Obese & $88(5.5)$ & $104(7.4)$ & $1(0.5)$ & $37(5.4)$ & $50(6.8)$ \\
\hline Missing & $391(24.3)$ & $375(26.8)$ & $56(30.1)$ & $172(25.2)$ & $163(22.1)$ \\
\hline Maternal smoking & $172(10.7)$ & $158(11.3)$ & $16(8.6)$ & $69(10.1)$ & $87(11.8)$ \\
\hline \multicolumn{6}{|l|}{ Maternal supplementation } \\
\hline Multivitamins & $201(12.5)$ & $166(11.9)$ & $33(17.7)$ & $105(15.4)$ & $63(8.5)$ \\
\hline Iron only & $498(31.0)$ & $456(32.6)$ & $44(23.7)$ & $203(29.8)$ & $251(34.0)$ \\
\hline Folic acid only & $13(0.8)$ & $20(1.4)$ & $2(1.1)$ & $7(1.0)$ & $4(0.5)$ \\
\hline Iron and folic acid & $142(8.8)$ & $104(7.4)$ & $8(4.3)$ & $52(7.6)$ & $82(11.1)$ \\
\hline None of the above & $750(46.7)$ & $653(46.7)$ & $98(52.7)$ & $315(46.2)$ & $337(45.6)$ \\
\hline $\begin{array}{l}\text { Maternal psychiatric } \\
\text { history }\end{array}$ & $550(34.2)$ & $694(49.6)$ & $53(28.5)$ & 229 (33.6) & $268(36.3)$ \\
\hline \multicolumn{6}{|l|}{ Maternal birth country } \\
\hline Nordic & $1243(77.3)$ & $1079(77.1)$ & $177(95.2)$ & $619(90.8)$ & $447(60.5)$ \\
\hline Africa & $90(5.6)$ & $97(6.9)$ & $0(0)$ & $4(0.6)$ & $86(11.6)$ \\
\hline Asia & $166(10.3)$ & $112(8.0)$ & $2(1.1)$ & $17(2.5)$ & $147(19.9)$ \\
\hline Other Europe & $66(4.1)$ & $57(4.1)$ & $4(2.2)$ & $26(3.8)$ & $36(4.9)$ \\
\hline Other & $42(2.6)$ & $54(3.9)$ & $3(1.6)$ & $16(2.3)$ & $23(3.1)$ \\
\hline
\end{tabular}

\section{Vitamin D measures}

25OHD concentrations by case status and maternal region of origin are summarized in Table 2. Median (IQR) values of standardized maternal and neonatal $25 \mathrm{OHD}$ were $57.1 \mathrm{nmol} /$ $\mathrm{L}(45.8,65.1)$ and $26.1(17.4,38.2)$, respectively. Of the mothers, $67.5 \%$ had sufficient $25 \mathrm{OHD}, 23.9 \%$ insufficient 25OHD, and $8.6 \%$ deficient 25OHD. Of the neonates, $10.0 \%$ had sufficient $25 \mathrm{OHD}, 41.2 \%$ insufficient 25OHD, and $48.7 \%$ deficient 25OHD. Maternal and neonatal were moderately correlated (Spearman correlations for maternal natural 25OHD and neonatal 25OHD: 0.50; and maternal standardized 25OHD and neonatal 25OHD: 0.45). Figure 1a shows a seasonal pattern such that higher 25OHD values are observed for summer births and lower values for winter births. 25OHD measures varied by maternal region of origin, such that Nordic-born mothers had the highest concentrations and Africa-born mothers the lowest (Fig. 1b).

\section{Maternal vitamin D and ASD}

For standardized maternal 25OHD, persons without ASD had median (IQR) concentrations of $56.1 \mathrm{nmol} / \mathrm{L}$ (35.1, 76.3), as compared to persons with ASD with ID [43.8 $\mathrm{nmol} / \mathrm{L}(19.7,63.8)]$ and ASD without ID [54.2 nmol/L (36.5, 69.7)] (Table 2, Fig. 1c). 
Table 2 Median (IQR) of 25OHD concentrations in $\mathrm{nmol} / \mathrm{L}$

\begin{tabular}{lclc}
\hline & $\begin{array}{l}\text { Maternal 25OHD } \\
\text { (natural) }\end{array}$ & $\begin{array}{l}\text { Maternal 25OHD (standardized } \\
\text { to } 10.9 \text { weeks gestation) }\end{array}$ & Neonatal 25OHD \\
\hline Entire sample & $54.2(33.9,73.1)$ & $57.1(45.8,65.1)$ & $26.1(17.4,38.2)$ \\
Nordic-born mothers & $59.5(45.0,77.4)$ & $60.0(53.0,68.2)$ & $29.3(20.8,41.2)$ \\
Non-Nordic born mothers & $26.7(15.6,44.2)$ & $30.8(22.7,45.1)$ & $15.1(9.8,23.0)$ \\
By outcome status (entire sample) & & $25.7(17.5,37.6)$ \\
ASD & $50.2(32.6,68.4)$ & $55.1(45.7,64.0)$ & $22.7(14.3,34.1)$ \\
ASD with ID & $43.8(19.7,63.8)$ & $49.7(28.7,63.2)$ & $26.9(18.8,39.2)$ \\
ASD without ID & $54.2(36.5,69.7)$ & $57.2(49.0,64.2)$ & $26.6(17.3,38.6)$ \\
No ASD & $56.1(35.1,76.3)$ & $58.1(46.3,66.5)$ & $28.9(20.6,40.7)$ \\
By outcome status (Nordic-born mothers) & & $29.3(20.7,41.0)$ \\
ASD & $56.4(42.5,73.7)$ & $58.9(51.7,66.9)$ & $28.7(20.6,40.5)$ \\
ASD with ID & $57.7(43.8,73.8)$ & $59.8(51.7,68.5)$ & $29.7(21.0,42.2)$ \\
ASD without ID & $56.1(42.4,73.3)$ & $58.8(51.8,65.7)$ & \\
No ASD & $62.3(47.6,79.6)$ & $60.8(54.3,68.7)$ & \\
\hline
\end{tabular}

Number of maternal samples $=1023$; Number of neonatal samples $=3006$
In adjusted models, there were no associations of maternal 25OHD with child ASD in the total sample (Table 3). In the subsample of participants with Nordic-born mothers, those with insufficient maternal 25OHD had 1.58 times higher odds of child ASD than those with sufficient 25OHD. This association in the Nordic subsample was most prominent for ASD with ID, where 25OHD insufficiency was associated with 2.35 times higher odds of the outcome compared with 25OHD sufficiency. In a model with linear parameterization of $25 \mathrm{OHD}$, each $25 \mathrm{nmol} / \mathrm{L}$ increase in maternal 25OHD was associated with 0.52 times lower odds of ASD with ID (Table 3). Generalized additive models were consistent with the linear parameterization, indicating a monotonic protective trend (Fig. S4). In sensitivity analyses of the $78 \%$ of participants with maternal $25 \mathrm{OHD}$ values measured in the 1st trimester, conclusions based on measured 25OHD values were broadly consistent with conclusions based on the standardized 25OHD values (Table S3).

\section{Neonatal vitamin D and ASD}

For neonatal 25OHD, persons without ASD had median (IQR) concentrations of $26.6 \mathrm{nmol} / \mathrm{L}(17.3,38.6)$, as compared with persons with ASD with ID [22.7 nmol/L (14.3, $34.1)]$ and persons with ASD without ID [26.9 nmol/L $(18.8,39.2)]$ (Table 2 Fig. 1c).

In adjusted models, compared with neonates with $25 \mathrm{OHD} \geq 50 \mathrm{nmol} / \mathrm{L}$, those with $25 \mathrm{OHD}<25 \mathrm{nmol} / \mathrm{L}$ had 1.33 times higher odds of ASD (Table 3). These associations were present for ASD without ID (1.43 times higher odds for $<25$ vs. $\geq 50 \mathrm{nmol} / \mathrm{L}$ ) but not for ASD with ID. These results were also observed in the Nordic subsample. In a linear parameterization of $25 \mathrm{OHD}$, an increase of 25 $\mathrm{nmol} / \mathrm{L}$ was associated with 0.86 times the odds of ASD. Analyses of the matched-sibling sample (Fig. S4c) were similar but with reduced precision (OR and $95 \%$ CI per 1 SD linear increase in 25OHD: ASD: 0.86 [0.73, 1.01]; ASD without ID: 0.86 [0.69, 1.06]; ASD with ID: 0.88 [0.66, $1.16]$ ), indicating that the results were unlikely to be due to familial confounding.

\section{Maternal and neonatal vitamin D and ASD}

In adjusted analyses, having maternal and neonatal 25OHD values below the median was associated with elevated odds of ASD and ASD without ID as compared with having maternal and neonatal 25OHD both above the median (Table 4). Individuals with both maternal and neonatal 25OHD concentrations below the median had 1.75 times (95\% CI: 1.08, 2.86) higher odds of ASD than persons with both maternal and neonatal 25OHD above the medians. Adjustment for maternal 25OHD did not influence the associations of neonatal 25OHD and ASD (Table S4), suggesting that the maternal and neonatal associations were largely independent of each other.

\section{Discussion}

The present study in a Swedish population-based cohort examined vitamin $\mathrm{D}$ in prospectively collected maternal and neonatal blood samples and risk of ASD. Our results suggest that higher concentrations of 25OHD were associated with a modestly lower risk of ASD. In the overall sample, maternal 25OHD was not associated with ASD. However, in participants with Nordic mothers, higher maternal 25OHD was associated with lower odds of ASD. In 
Fig. 1 Neonatal and maternal (natural) 25OHD concentrations by time of sampling (a), maternal region of origin (b), and ASD case status (c)
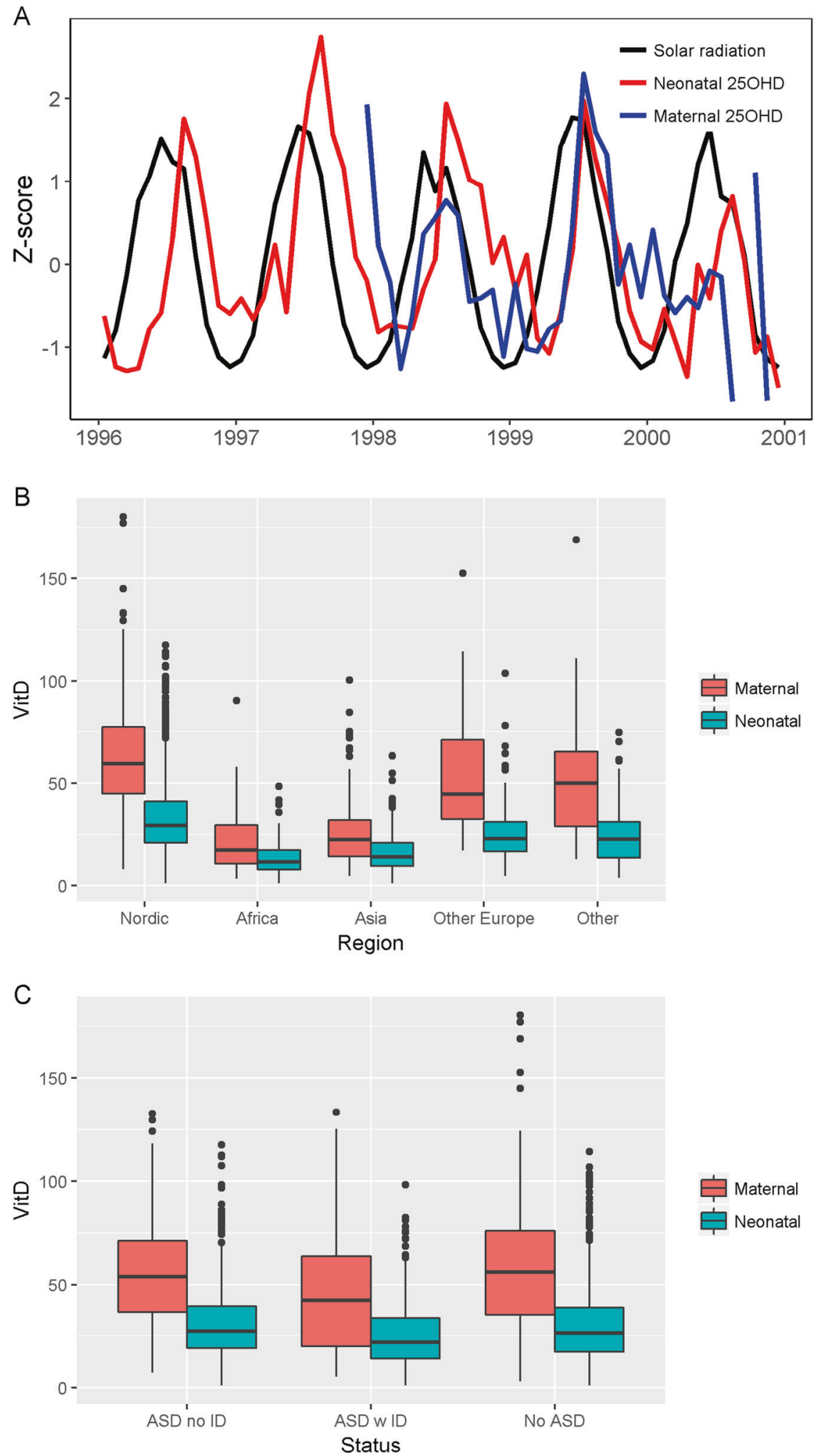

analyses subtyped by co-occurring ID, higher maternal 25OHD was more associated with lower odds of ASD with ID, whereas higher neonatal 25OHD was more associated with lower odds of ASD without ID.

\section{Strengths and weaknesses}

The present study features the largest number of ASD cases in a study of early life vitamin D and ASD. Vitamin D 
Table 3 Associations of 25OHD concentrations and ASD in the Stockholm Youth Cohort

\begin{tabular}{|c|c|c|c|c|}
\hline & \multicolumn{2}{|l|}{ Maternal $25 \mathrm{OHD}^{\mathrm{a}}$} & \multicolumn{2}{|l|}{ Neonatal 25OHD } \\
\hline & $\begin{array}{l}\text { Total sample: Number of } \\
\text { exposed cases/odds ratio } \\
(95 \% \mathrm{CI})\end{array}$ & $\begin{array}{l}\text { Nordic }^{\mathrm{b}}: \text { Number of } \\
\text { exposed cases/odds ratio } \\
(95 \% \text { CI })\end{array}$ & $\begin{array}{l}\text { Total sample: Number of } \\
\text { exposed cases/odds ratio } \\
(95 \% \mathrm{CI})\end{array}$ & $\begin{array}{l}\text { Nordic: Number of exposed } \\
\text { cases/odds ratio }(95 \% \mathrm{CI})\end{array}$ \\
\hline \multicolumn{5}{|l|}{$\underline{\mathrm{ASD}}$} \\
\hline$<25 \mathrm{nmol} / \mathrm{L} 25 \mathrm{OHD}$ & $46 / 1.67(0.76,3.67)$ & - & 676/1.33 (1.01, 1.74) & 421/1.40 (1.04, 1.87) \\
\hline $25-<50 \mathrm{nmol} / \mathrm{L}$ & $114 / 1.19(0.79,1.78)$ & 67/1.58 (1.00, 2.49) & $573 / 1.11(0.86,1.43)$ & $516 / 1.13(0.86,1.47)$ \\
\hline$\geq 50 \mathrm{nmol} / \mathrm{L}$ & 289/Reference & 261/Reference & 150/Reference & 142/Reference \\
\hline $\begin{array}{l}\text { 25OHD increase of } 25 \\
\mathrm{nmol} / \mathrm{L}^{\mathrm{c}}\end{array}$ & $0.84(0.60,1.16)$ & $0.65(0.43,0.96)$ & $0.90(0.78,1.02)$ & $0.86(0.74,0.99)$ \\
\hline \multicolumn{5}{|l|}{ ASD without ID } \\
\hline$<25 \mathrm{nmol} / \mathrm{L} 25 \mathrm{OHD}$ & $14 / 1.83(0.64,5.31)$ & - & 428/1.44 (1.06, 1.96) & 318/1.48 (1.07, 2.05) \\
\hline $25-<50 \mathrm{nmol} / \mathrm{L}$ & $67 / 1.25(0.79,1.97)$ & $48 / 1.47(0.89,2.44)$ & $412 / 1.14(0.86,1.52)$ & 383/1.16 (0.87, 2.05) \\
\hline$\geq 50 \mathrm{nmol} / \mathrm{L}$ & 213/Reference & 197/Reference & 107/Reference & 103/Reference \\
\hline $\begin{array}{l}\text { 25OHD increase of } 25 \\
\mathrm{nnmol} / \mathrm{L}\end{array}$ & $0.80(0.54,1.17)$ & $0.67(0.43,1.03)$ & $0.90(0.77,1.04)$ & $0.85(0.73,1.00)$ \\
\hline \multicolumn{5}{|l|}{ ASD with ID } \\
\hline$<25 \mathrm{nmol} / \mathrm{L} 25 \mathrm{OHD}$ & $32 / 1.62(0.59,4.44)$ & - & 248/1.08 (0.72, 1.64) & $103 / 1.15(0.73,1.82)$ \\
\hline $25-<50 \mathrm{nmol} / \mathrm{L}$ & $47 / 1.25(0.67,2.29)$ & 19/2.35 (1.14, 4.79) & $161 / 1.00(0.69,1.49)$ & $133 / 1.00(0.67,1.53)$ \\
\hline$\geq 50 \mathrm{nmol} / \mathrm{L}$ & 76/Reference & 64/Reference & 43/Reference & 39/Reference \\
\hline $\begin{array}{l}\text { 25OHD increase of } 25 \\
\mathrm{nmol} / \mathrm{L}\end{array}$ & $0.90(0.54,1.48)$ & $0.52(0.27,0.98)$ & $0.90(0.73,1.10)$ & $0.87(0.68,1.10)$ \\
\hline
\end{tabular}

Estimates are derived from logistic regression models adjusted for year of birth, sample month, and maternal characteristics: psychiatric disorders, age, body mass index, smoking, nutritional supplement use, and region of origin

${ }^{a}$ Maternal 25OHD values are estimated from a prediction model that corrects for different gestational ages at sampling

${ }^{b}$ Estimates for the subset of children born to Nordic mothers are adjusted for all of above except for maternal region of origin

${ }^{c}$ Odds ratio associated with a linear increase of $25 \mathrm{nmol} / \mathrm{L}$ in maternal or neonatal $25 \mathrm{OHD}$

levels were measured from both maternal and neonatal blood, thus increasing the likelihood that the study reflects biological processes occurring during a temporal window of etiological relevance. The ASD case finding approach has been previously validated and occurred in the context of a universal healthcare system, increasing the likelihood that cases were identified. All study data were prospectively assessed, minimizing the potential for recall bias to influence results.

Nutritional epidemiology studies are often susceptible to confounding [41], since it is likely that nutritional patterns co-vary with other characteristics (e.g., healthful behaviors) that may influence risk of the outcome. Our study found that multiple characteristics differed between persons of different vitamin D status. Although our statistical models adjusted for a wide range of relevant covariates, results may still be influenced by confounding. The sibling analysis attempts to address this, through comparison of siblings who share early life environment and genetic background to a greater extent than unrelated individuals. Although our sibling analysis results did not reach conventional statistical significance, the point estimates were quite similar to our complete sample results, suggesting that familial environmental or genetic factors were unlikely to be responsible for observed associations. Still, confounding may be possible from a number of sources which could not be accounted for in the present study. For example, we did not measure concentrations of other nutrients in the neonatal blood samples, and it is possible that another nutrient that is highly correlated with vitamin D may in fact be responsible for the observed reduction in risk that is attributed to higher vitamin D concentrations. We previously reported that multivitamin supplement use at first antenatal visit was associated with reduced risk of ASD with ID [4], and while persons with multivitamin use had higher 25OHD concentrations, other nutrients are also likely elevated in such supplement users.

Another limitation of the present study is that it features a sample based in Sweden, thereby limiting the generalizability of results to other populations. Since vitamin D is influenced by sunlight exposure, the latitude of the study setting affects the possible exposure range of the study sample. We did not have adequate power to study the outcome of ASD among non-Nordic born mothers, which precluded any further analysis according to maternal region of origin. Our group has previously noted an elevated risk 
Table 4 Associations of maternal and neonatal 25OHD and ASD by presence or absence of intellectual disability in the Stockholm Youth Cohort. N of exposed ASD cases in each strata of analysis / odds ratio ( $95 \%$ confidence interval)

\begin{tabular}{|c|c|c|}
\hline Outcome & Total sample $n=766$ & Nordic mothers $^{\mathrm{a}} n=573$ \\
\hline \multicolumn{3}{|l|}{$\underline{\mathrm{ASD}}$} \\
\hline Maternal below median, neonatal below median & 131/1.75 (1.08, 2.86) & $57 / 1.94(\mathbf{1 . 1 1}, \mathbf{3 . 4 1})$ \\
\hline Maternal below median, neonatal above median & $60 / 1.48(0.92,2.40)$ & $51 / 1.78(\mathbf{1 . 0 4}, \mathbf{3 . 0 6})$ \\
\hline Maternal above median, neonatal below median & $51 / 1.17(0.73,1.89)$ & $46 / 1.04(0.62,1.75)$ \\
\hline Maternal above median, neonatal above median & 98/Ref & 93/Ref \\
\hline \multicolumn{3}{|l|}{ ASD without ID } \\
\hline Maternal below median, neonatal below median & 73/2.13 (1.24, 3.70) & 46/1.99 (1.09, 3.63) \\
\hline Maternal below median, neonatal above median & $40 / 1.50(0.86,2.59)$ & $38 / 1.64(0.91,2.96)$ \\
\hline Maternal above median, neonatal below median & $39 / 1.11(0.65,1.89)$ & $36 / 1.02(0.57,1.78)$ \\
\hline Maternal above median, neonatal above median & 74/Ref & 72/Ref \\
\hline \multicolumn{3}{|l|}{ ASD with ID } \\
\hline Maternal below median, neonatal below median & $58 / 1.24(0.56,2.72)$ & $11 / 2.03(0.76,5.40)$ \\
\hline Maternal below median, neonatal above median & $20 / 1.46(0.68,3.08)$ & $13 / 2.40(0.95,6.05)$ \\
\hline Maternal above median, neonatal below median & $12 / 1.20(0.52,2.65)$ & $10 / 0.83(0.32,2.11)$ \\
\hline Maternal above median, neonatal above median & 24/Ref & $21 / \operatorname{Ref}$ \\
\hline
\end{tabular}

of ASD with ID for individuals born to mothers from SubSaharan and Northern Africa, Latin America, the Caribbean, and Southern Asia [42]. While non-Nordic born mothers and their children had notably lower levels of 25OHD compared with Nordic-born mothers, there are also complex cultural, environmental, and diagnostic factors that may be responsible for potential ASD risk differences between Nordic and non-Nordic groups in Sweden. This is also related to the next limitation of our study: although generalized additive models suggested a monotonically linear association between higher 25OHD and lower ASD risk, there were relatively few persons at the extreme values of 25OHD. This hinders examination of the upper range of values and potential thresholds of risk. For example, it is possible that increasing levels of $25 \mathrm{OHD}$ beyond a certain point may not actually further reduce ASD risk, but our study is unable to address such questions.

Caution must also be used in interpreting the cut-offs used in this study to denote deficiency and insufficiency. These cut-offs were set by the Institute of Medicine in reference to serum measurements in adults, while our study includes neonates and extrapolates serum equivalents from the concentrations measured in DBS. It is possible that different standards of deficiency and insufficiency should be applied to neonates compared to adults, although this issue has not been well studied. We observed that neonatal 25OHD levels were considerably lower than maternal levels measured during pregnancy. This is in line with longstanding observations that maternal 25OHD levels tend to be higher than, though positively correlated with, neonatal levels [43], as well as with recent observations from large cohort studies [13]. Previous studies using paired samples from mothers and their newborn children indicate that neonatal levels are on average lower than maternal levels and the prevalence of insufficiency is thus higher among neonates when the same cut-offs are used [21].

\section{Interpretation}

Existing studies on the topic of 25OHD and ASD are few in number. Our findings agree with some of the existing literature but disagree with others. A recent analysis of birth trends of ASD cases in five countries found evidence for seasonality in Sweden and Finland. Specifically, the analysis found that children born in the fall months (i.e., conceived in the winter) had the highest risk of ASD and those born in the spring months (i.e., conceived in the summer) had the lowest risk of ASD [44]. Our findings that higher concentrations of perinatal/neonatal 25OHD are associated with lower risk of ASD is consistent with prior studies in the Netherlands [18, 21] and China [45], but disagree with overall null associations found in two studies from California [22, 23]. Laboratory differences are not a factor since the same laboratory was used for our study and the two California studies (the Early Markers for Autism (EMA) study and the CHARGE study). The California studies featured substantially higher vitamin D levels than in our study, as would be expected according to the geographical 
and sunlight differences between California and Sweden, and as such may explain differences in observed results. The EMA sample had a median neonatal 25OHD concentration of $84 \mathrm{nmol} / \mathrm{L}$ (controls); the CHARGE sample had a mean neonatal $25 \mathrm{OHD}$ concentration of $79.6 \mathrm{nmol} / \mathrm{L}$; while our study had a median of $26.1 \mathrm{nmol} / \mathrm{L}$. If there is a threshold effect of vitamin D on ASD risk, overall high levels in a sample could potentially mask such an effect. However, the California studies found racial/ethnic differences, with protective associations of higher 25OHD suggested for either ASD or developmental delay, but only in non-Hispanic whites. In our study, we assessed associations of 25OHD and ASD in analyses stratified by maternal origin, given the well-known differences by race/ethnicity in 25OHD. Consistent with the aforementioned findings above, evidence of a 25OHD-ASD association was observed in the subsample with mothers born in Nordic countries, a region that is generally associated with having lighter skin pigmentation. Even though our study was relatively large, the sample primarily consisted of mothers from Nordic countries. We had neither skin pigmentation data nor the statistical power to be able to stratify more finely by maternal origin to examine these differences further.

The observation that higher maternal 25OHD was more protective for ASD with ID (the more severe phenotype) but that higher neonatal 25OHD was more protective for ASD without ID, raises interesting questions about timing and critical windows for neurodevelopment. In the paired maternal-neonatal sample, we observed the lowest ASD risk for persons with both maternal and neonatal 25OHD above the median, and the highest risk for the group with both maternal and neonatal 25OHD below the median. Persons with maternal 25OHD below the median but neonatal 25OHD above the median had still elevated, but somewhat reduced risk, as compared with persons with both low maternal and neonatal 25OHD. Since fetal 25OHD is largely dependent on maternal levels [46], these results suggest that the ASD risk due to low 25OHD in early gestation may be slightly mitigated by increased 25OHD in later gestation. Therefore, 25OHD may have more substantial influence on ASD risk earlier in gestation as opposed to later, with lower levels earlier on increasing risk for a more severe phenotype of ASD with ID, and lower levels later on increasing risk for the less severe phenotype of ASD without ID. Only a few studies have examined critical windows for vitamin D for neurodevelopment. In experimental studies using rats, developmental vitamin D deficiency is known to result in enlarged lateral ventricles in neonates [47]. However, adding vitamin D back into the diet at birth partially ameliorated these structural changes [48]. O'Loan et al. compared behavioral phenotypes of rats exposed to different periods of vitamin D deficiency during gestation [49]. Rats exposed to late deficiency (a vitamin Ddeficient maternal diet during pregnancy), and full deficiency (a vitamin D-deficient maternal diet both preconception and during pregnancy) exhibited increased hyperlocomotion in response to treatment with the NDMAreceptor antagonist MK-801. Interestingly, rats with early deficiency (a vitamin D-deficient maternal diet pre-conception, but with a replete diet during pregnancy) did not exhibit such abnormal behavior, suggesting that prompt prenatal intervention may reduce adverse outcomes.

It should be noted that although we found that 25OHD was associated with ASD in general regardless of cooccurring ID, it may be possible that 25OHD influences neurodevelopmental processes in a way that is not specific to ASD. A recent meta-analysis suggests that increased prenatal 25OHD is associated with better cognitive development and lower risk of ADHD [50]. Lower neonatal vitamin $\mathrm{D}$ status has also been associated with increased odds of schizophrenia [29]. There is robust evidence from animal studies that vitamin D is involved in a range of processes relevant to the developing brain, including cell differentiation, cytokine regulation, neurotransmitter synthesis, calcium signaling, anti-oxidant activity, and gene/protein expression governing neuronal physiology [51]. Thus, it is plausible that vitamin D may influence risk of ASD and other neurodevelopmental conditions through any of these mechanisms. Increasing evidence suggests that the placenta is instrumental in mediating the manifold effects of vitamin D [46]. The placenta expresses the components necessary for vitamin D signaling including the vitamin $\mathrm{D}$ receptor (VDR), retinoid $\mathrm{X}$ receptor (RXR), and the cytochrome P450 enzymes CYP27B1 and CYP24A1. Vitamin D has immunomodulatory properties, generally reducing adaptive immune activity and promoting innate immune activity [52]. Epidemiological studies have shown that maternal hospitalization with infection during pregnancy is associated with increased risk of ASD in offspring [53, 54]. This is consistent with animal models of maternal immune activation that show ASD-related phenotypes resulting from maternal injection with lipopolysaccharide (as a bacterial infection analogue) or poly(I:C) (as a viral infection analogue) [55]. Interestingly, administration of calcitriol to poly(I:C)-exposed pregnant mice prevents the ASD-related phenotypes in offspring [56]. In rats, challenge of vitamin D-deficient placentas with poly(I: C) resulted in higher production of IL-6 and IL-1B in male fetuses [57]. In humans, elevated levels of maternal IL-6 [58] and neonatal IL-1B [59] have been associated with increased ASD risk. Further studies are required to understand if the influence of early life vitamin D exposure on maternal-fetal immune responses constitutes a plausible mechanism linking early life vitamin D exposure to risk for ASD in humans. 


\section{Conclusions}

Even with mounting epidemiological evidence from our study and others linking early life vitamin D to ASD, it is premature to conclude that a causal link exists, since all studies to date have been observational in nature. Ethically designed randomized controlled trials of vitamin D supplementation during pregnancy would allow for a clear answer.

Acknowledgements This study is supported by National Institutes of Health grant no. NIH 1 R21 ES023760-01A1. JM is supported by the Danish National Research Foundation (Niels Bohr Professorship to John McGrath) and a John Cade Fellowship (APP1056929) from National Health and Medical Research Council. RG is supported by the Swedish Research Council (grant number 2017-02900).

\section{Compliance with ethical standards}

Conflict of interest The authors declare that they have no conflict of interest.

Publisher's note Springer Nature remains neutral with regard to jurisdictional claims in published maps and institutional affiliations.

Open Access This article is licensed under a Creative Commons Attribution 4.0 International License, which permits use, sharing, adaptation, distribution and reproduction in any medium or format, as long as you give appropriate credit to the original author(s) and the source, provide a link to the Creative Commons license, and indicate if changes were made. The images or other third party material in this article are included in the article's Creative Commons license, unless indicated otherwise in a credit line to the material. If material is not included in the article's Creative Commons license and your intended use is not permitted by statutory regulation or exceeds the permitted use, you will need to obtain permission directly from the copyright holder. To view a copy of this license, visit http://creativecommons. org/licenses/by/4.0/.

\section{References}

1. Prado EL, Dewey KG. Nutrition and brain development in early life. Nutr Rev. 2014;72:267-84.

2. Lyall K, Croen L, Daniels J, Fallin MD, Ladd-Acosta C, Lee BK, et al. The changing epidemiology of autism spectrum disorders. Annual Rev Public Health. 2017;38:81-102.

3. Suren P, Roth C, Bresnahan M, Haugen M, Hornig M, Hirtz D, et al. Association between maternal use of folic acid supplements and risk of autism spectrum disorders in children. J Am Med Assoc. 2013;309:570-7.

4. DeVilbiss EA, Magnusson C, Gardner RM, Rai D, Newschaffer $\mathrm{CJ}$, Lyall $\mathrm{K}$, et al. Antenatal nutritional supplementation and autism spectrum disorders in the Stockholm youth cohort: population based cohort study. BMJ. 2017;359:j4273.

5. Lyall K, Munger KL, O'reilly ÉJ, Santangelo SL, Ascherio A. Maternal dietary fat intake in association with autism spectrum disorders. Am J Epidemiol. 2013;178:209-20.

6. Schmidt RJ, Tancredi DJ, Krakowiak P, Hansen RL, Ozonoff S. Maternal intake of supplemental iron and risk of autism spectrum disorder. Am J Epidemiol. 2014;180:890-900.

7. Strom M, Granstrom C, Lyall K, Ascherio A, Olsen SF. Research Letter: Folic acid supplementation and intake of folate in pregnancy in relation to offspring risk of autism spectrum disorder. Psychol Med 2018;48:1048-54.

8. Virk J, Liew Z, Olsen J, Nohr EA, Catov JM, Ritz B. Preconceptional and prenatal supplementary folic acid and multivitamin intake and autism spectrum disorders. Autism. 2016;20:710-8.

9. Kocovska E, Fernell E, Billstedt E, Minnis H, Gillberg C. Vitamin D and autism: clinical review. Res Dev Disabil. 2012;33:1541-50.

10. Eyles D, Burne T, McGrath J. Vitamin D in fetal brain development. Semin Cell Dev Biol. 2011;22:629-36.

11. Holick MF. Vitamin D deficiency. N Engl j Med. 2007; 2007:266-81.

12. Ginde AA, Sullivan AF, Mansbach JM, Camargo CA. Vitamin D insufficiency in pregnant and nonpregnant women of childbearing age in the United States. Am J Obstet Gynecol. 2010;202:436. e431-6. e438.

13. Vinkhuyzen AAE, Eyles DW, Burne TH, Blanken LME, Kruithof CJ, Verhulst F, et al. Prevalence and predictors of vitamin D deficiency based on maternal mid-gestation and neonatal cord bloods: the generation R study. J Steroid Biochem Mol Biol. 2016;164:161-7.

14. Holick MF, Chen TC. Vitamin D deficiency: a worldwide problem with health consequences. Am J Clin Nutr. 2008;87: 1080S-6S.

15. Bodnar LM, Simhan HN, Powers RW, Frank MP, Cooperstein E, Roberts JM. High prevalence of vitamin D insufficiency in black and white pregnant women residing in the northern United States and their neonates. J Nutr. 2007;137:447-52.

16. Whitehouse AJ, Holt BJ, Serralha M, Holt PG, Hart PH, Kusel MM. Maternal vitamin D levels and the autism phenotype among offspring. J Autism Dev Disord. 2013;43:1495-1504.

17. Whitehouse AJ, Holt BJ, Serralha M, Holt PG, Kusel MM, Hart $\mathrm{PH}$. Maternal serum vitamin D levels during pregnancy and offspring neurocognitive development. Pediatrics. 2012;129:485-93.

18. Vinkhuyzen AA, Eyles D, Burne TH, Blanken LM, Kruithof CJ, Verhulst F, et al. Gestational vitamin D deficiency and autismrelated traits: the Generation R Study. Mol Psychiatry. 2018; 23:240.

19. Fernell E, Barnevik-Olsson M, Bagenholm G, Gillberg C, Gustafsson S, Saaf M. Serum levels of 25-hydroxyvitamin D in mothers of Swedish and of Somali origin who have children with and without autism. Acta Paediatr. 2010;99:743-7.

20. Chen J, Xin K, Wei J, Zhang K, Xiao H. Lower maternal serum $25(\mathrm{OH}) \mathrm{D}$ in first trimester associated with higher autism risk in Chinese offspring. J Psychosom Res. 2016;89:98-101.

21. Vinkhuyzen AA, Eyles DW, Burne TH, Blanken LM, Kruithof CJ, Verhulst F, et al. Gestational vitamin D deficiency and autism spectrum disorder. Br J Psychiatry Open. 2017;3:85-90.

22. Windham GC, Pearl M, Anderson MC, Poon V, Eyles D, Jones $\mathrm{KL}$, et al. Newborn vitamin D levels in relation to autism spectrum disorders and intellectual disability: a case-control study in California. Autism Res. 2019;12:989-98.

23. Schmidt RJ, Niu Q, Eyles DW, Hansen RL, Iosif AM. Neonatal vitamin $\mathrm{D}$ status in relation to autism spectrum disorder and developmental delay in the CHARGE case-control study. Autism Res. 2019;12:976-88.

24. Idring S, Rai D, Dal H, Dalman C, Sturm H, Zander E, et al Autism spectrum disorders in the Stockholm youth cohort: design, prevalence and validity. PloS ONE. 2012;7:e41280.

25. Eyles D, Anderson C, Ko P, Jones A, Thomas A, Burne T, et al. A sensitive LC/MS/MS assay of $25 \mathrm{OH}$ vitamin D3 and $25 \mathrm{OH}$ vitamin D2 in dried blood spots. Clin Chim Acta. 2009; 403:145-51.

26. Lawlor DA, Wills AK, Fraser A, Sayers A, Fraser WD, Tobias $\mathrm{JH}$. Association of maternal vitamin D status during pregnancy 
with bone-mineral content in offspring: a prospective cohort study. Lancet. 2013;381:2176-83.

27. Kvaskoff D, Heath AK, Simila HA, Ko P, English DR, Eyles DW. Minimizing matrix effects for the accurate quantification of 25Hydroxyvitamin D metabolites in dried blood spots by LC-MS/ MS. Clin Chem. 2016;62:639-46.

28. Fernell E, Bejerot S, Westerlund J, Miniscalco C, Simila H, Eyles D, et al. Autism spectrum disorder and low vitamin D at birth: a sibling control study. Mol Autism. 2015;6:3.

29. McGrath JJ, Eyles DW, Pedersen CB, Anderson C, Ko P, Burne $\mathrm{TH}$, et al. Neonatal vitamin D status and risk of schizophrenia: a population-based case-control study. Arch Gen Psychiatry. 2010;67:889-94.

30. Kvaskoff D, Ko P, Simila HA, Eyles DW. Distribution of 25hydroxyvitamin D3 in dried blood spots and implications for its quantitation by tandem mass spectrometry. J Chromatogr B. 2012;901:47-52.

31. Heath AK, Williamson EJ, Ebeling PR, Kvaskoff D, Eyles DW, English DR. Measurements of 25-hydroxyvitamin D concentrations in archived dried blood spots are reliable and accurately reflect those in plasma. J Clin Endocrinol Metab. 2014;99:3319-24.

32. Bates I. Reference ranges and normal values, Dacie and Lewis Practical Haematology. Edinburgh: Churchill Livingstone Elsevier; 2012.

33. Stackhouse PW Jr, Gupta SK, Cox SJ, Zhang T, Mikovitz JC, Hinkelman LM. The NASA/GEWEX surface radiation budgetrelease 3.0: 24.5-year dataset. GEWEX News. 2011;21:10-2.

34. Idring S, Lundberg M, Sturm H, Dalman C, Gumpert C, Rai D, et al. Changes in prevalence of autism spectrum disorders in 2001-11: findings from the stockholm youth cohort. J Autism Dev Disord. 2015;45:1766-73.

35. Rai D, Lee BK, Dalman C, Golding J, Lewis G, Magnusson C. Parental depression, maternal antidepressant use during pregnancy, and risk of autism spectrum disorders: population based case-control study. BMJ. 2013;346:f2059.

36. Gardner RM, Lee BK, Magnusson C, Rai D, Frisell T, Karlsson $\mathrm{H}$, et al. Maternal body mass index during early pregnancy, gestational weight gain, and risk of autism spectrum disorders: results from a Swedish total population and discordant sibling study. Int J Epidemiol. 2015;44:870-83.

37. Lee BK, Gardner RM, Dal H, Svensson A, Galanti MR, Rai D, et al. Brief report: maternal smoking during pregnancy and autism spectrum disorders. J Autism Dev Disord. 2012;42: 2000-5.

38. Xie S, Heuvelman H, Magnusson C, Rai D, Lyall K, Newschaffer $\mathrm{CJ}$, et al. Prevalence of autism spectrum disorders with and without intellectual disability by gestational age at birth in the stockholm youth cohort: a register linkage study. Paediatr Perinatal Epidemiol. 2017;31:586-94.

39. Ross AC, Taylor CL, Yaktine AL, Del Valle HB. Institute of medicine (US) committee to review dietary reference intakes for vitamin D and calcium. Dietary Reference Intakes for Calcium and Vitamin D. Washington (DC): National Academies Press; 2011. http://www.ncbi.nlm.nih.gov/books/NBK56056/. Accessed 02 Jan 2016.

40. Team RC. R: A language and environment for statistical computing. Vienna, Austria: Computing RFfS; 2017.

41. DeVilbiss EA, Gardner RM, Newschaffer CJ, Lee BK. Maternal folate status as a risk factor for autism spectrum disorders: a review of existing evidence. Br J Nutr. 2015;114:663-72.
42. Magnusson C, Rai D, Goodman A, Lundberg M, Idring S, Svensson A, et al. Migration and autism spectrum disorder: population-based study. Br J Psychiatry. 2012;201:109-15.

43. Paulson SK, DeLuca HF. Vitamin D metabolism during pregnancy. Bone. 1986;7:331-6.

44. Lee BK, Gross R, Francis RW, Karlsson H, Schendel DE, Sourander $\mathrm{A}$, et al. Birth seasonality and risk of autism spectrum disorder. Eur J Epidemiol. 2019;34:785-92.

45. Wu DM, Wen X, Han XR, Wang S, Wang YJ, Shen M, et al. Relationship between neonatal vitamin $\mathrm{D}$ at birth and risk of autism spectrum disorders: the NBSIB study. J Bone Min Res. 2018;33:458-66.

46. Karras SN, Wagner CL, Castracane VD. Understanding vitamin D metabolism in pregnancy: from physiology to pathophysiology and clinical outcomes. Metabolism. 2018;86:112-23.

47. Eyles D, Brown J, Mackay-Sim A, McGrath J, Feron F. Vitamin D3 and brain development. Neuroscience. 2003;118:641-53.

48. Feron F, Burne T, Brown J, Smith E, McGrath J, Mackay-Sim A, et al. Developmental vitamin D3 deficiency alters the adult rat brain. Brain Res Bull. 2005;65:141-8.

49. O'Loan J, Eyles DW, Kesby J, Ko P, McGrath JJ, Burne TH Vitamin D deficiency during various stages of pregnancy in the rat; its impact on development and behaviour in adult offspring. Psychoneuroendocrinology. 2007;32:227-34.

50. Garcia-Serna AM, Morales E. Neurodevelopmental effects of prenatal vitamin D in humans: systematic review and metaanalysis. Mol Psychiatry. 2019. https://doi.org/10.1038/s41380019-0357-9 [Epub ahead of print].

51. Eyles DW, Burne TH, McGrath JJ. Vitamin D, effects on brain development, adult brain function and the links between low levels of vitamin D and neuropsychiatric disease. Front Neuroendocrinol. 2013;34:47-64.

52. Adams JS, Hewison M. Unexpected actions of vitamin D: new perspectives on the regulation of innate and adaptive immunity. Nat Clin Pr Endocrinol Metab. 2008;4:80-90.

53. Lee BK, Magnusson C, Gardner RM, Blomström Å, Newschaffer CJ, Burstyn I, et al. Maternal hospitalization with infection during pregnancy and risk of autism spectrum disorders. Brain Behav Immun. 2015;44:100-5.

54. Atladottir HO, Thorsen P, Ostergaard L, Schendel DE, Lemcke S, Abdallah $\mathrm{M}$, et al. Maternal infection requiring hospitalization during pregnancy and autism spectrum disorders. J Autism Dev Disord. 2010;40:1423-30.

55. Boksa P. Effects of prenatal infection on brain development and behavior: a review of findings from animal models. Brain Behav Immun. 2010;24:881-97.

56. Vuillermot S, Luan W, Meyer U, Eyles D. Vitamin D treatment during pregnancy prevents autism-related phenotypes in a mouse model of maternal immune activation. Mol Autism. 2017;8:9.

57. Ali A, Cui X, Alexander S, Eyles D. The placental immune response is dysregulated developmentally vitamin $\mathrm{D}$ deficient rats: Relevance to autism. J Steroid Biochem Mol Biol. 2018; 180:73-80.

58. Jones KL, Croen LA, Yoshida CK, Heuer L, Hansen R, Zerbo O, et al. Autism with intellectual disability is associated with increased levels of maternal cytokines and chemokines during gestation. Mol Psychiatry. 2017;22:273.

59. Krakowiak P, Goines PE, Tancredi DJ, Ashwood P, Hansen RL, Hertz-Picciotto I, et al. Neonatal cytokine profiles associated with autism spectrum disorder. Biol Psychiatry. 2017;81:442-51. 